\title{
DESIGN ASPECTS OF INSTALLATION DEVICES OF THE SUPPORT RINGS
}

\author{
Alexei Botez*, ORCID ID: 0000-0001-8357-076X, \\ Elena Rusu, ORCID ID: 0000-0002-2473-0353 \\ Technical University of Moldova, 168 Stefan cel Mare Blvd, MD-2004, Chisinau, Republic of \\ *Corresponding author: Alexei Botez, alexei.botez@gddti.utm.md
}

Received: 06. 23. 2021

Accepted: 08. 11. 2021

\begin{abstract}
The processes of manufacturing machines and appliances show a continuous tendency to increase the degree of automation. An important role in the automation of manufacturing processes is played by the constructive technologicality of the product. It is important to use a minimum number of components, which have a construction as technological as possible in terms of automation. In this context, the use of supporting rings as fasteners is welcome. The design of the installation devices of these rings requires the knowledge of their deformation forces, information that is missing in the profile literature. The authors set their goal to develop the method for calculating the forces required to deform the supporting rings with a rectangular transverse profile and their maximum allowable deformations. The calculation relationships were obtained by formalizing the supporting ring through a bar with a fixed end and studying its deformation using Mohr's integral. The article lists some types of the supporting rings used in the construction of machines and appliances, their advantages and disadvantages, aspects of their automatic installation: deformation and installation methods, precision of joint orientation, optimal design, calculation of forces required for deformation and the maximum permissible deformation value. As a result of the research carried out, recommendations were developed regarding the calculation of some constructive parameters of the devices for installing the supporting rings.
\end{abstract}

Keywords: supporting ring, mounting process, deformation forces, accuracy of orientation.

\section{Introduction}

Assembly is the final stage, but no less important of the manufacturing process, which integrates in itself the results of the previous stages and outlines the quality of the final product. The share of assembly constitutes about $40 \%$ of the total volume of works performed in the manufacture of the product, $80 \%$ of the assembly operations remaining manual [1]. Some of the causes of the delayed development of the automation of the assembly processes being studied in [2] are:

- Lack of systematic research on assembly processes, leading to intuitive methods of automatic tool design [3];

- Constructive inconvenience of the product for automatic assembly [4]; 
- Problems, related to ensuring the accuracy of the mutual orientation of the assembled parts [5];

- $\quad$ The low degree of unification of the component parts of the product [6].

An important role in increasing the degree of automation of assembly is played by the quantity of component parts [7] and the technologicality of the assembled object [8].

It should be noted that about $20 \%$ of the total number of components of the machine is occupied by the fasteners. According to the number of components, the fasteners are divided into four groups [9]:

- Assemblies without fixing parts (gluing, welding, etc.);

- Assemblies with a fixing piece (fixing with support ring, pin, etc.);

- Assemblies with two fixing parts (screw and washer);

- Assemblies with three fixing parts (screw, washer, nut).

Practice dictates the use of fasteners as simple and technological as possible [10].

Thus, designers rightly use widely support rings as fasteners in their constructions.

They are part of the second group of fasteners - with one piece.

The advantages of using supporting rings are [11]:

- - Constructive improvement;

- $\quad$ - The possibility of using the assembly equipment relatively cheaply;

- - Optimization of technological manufacturing processes;

- - Optimal operating characteristics.

\section{Formulation of the Problem within the Study}

The supporting rings are usually made of wires or sheets of spring steels as the material.

The supporting rings can be of the following types:

- with round transverse profile (Figure 1a);

- with a rectangular cross-section (eccentric (Figure 1b, c) or concentric (Figure 1d));

- exterior (installed only on shafts, Figure 1c);

- interiors (installed only in holes, Figure 1b);

- universal (Figure 1d).

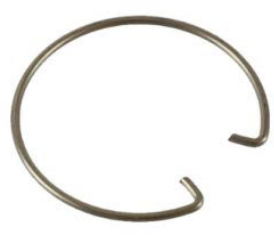

a)

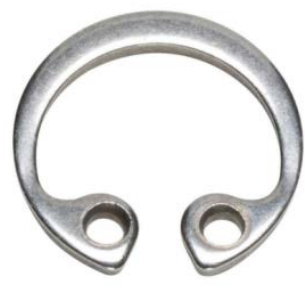

b)

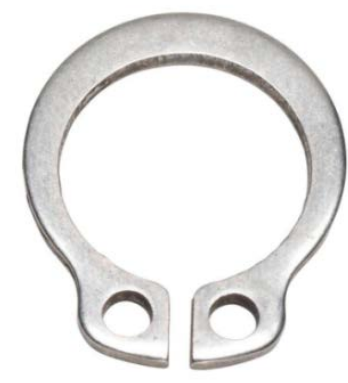

c)

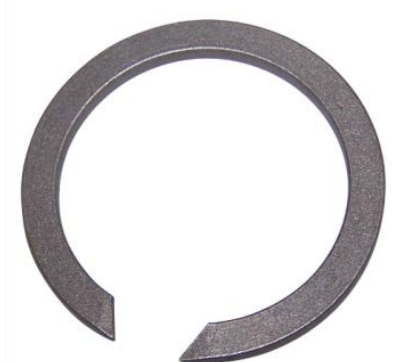

d)

Figure 1. Supporting rings.

Rings with round cross section are simple to manufacture, but their load capacity and positioning accuracy of fixed parts is low, therefore these are used only in places where they can meet its possibilities.

Rings with a rectangular transverse profile have better load-bearing and precision characteristics. In order to increase their deformability, they are made with eccentricity, but no information has been found in the literature to justify the value of eccentricity. 
The deformation methods are the following:

- by applying a concentrated force, deposited at the ends of the ring (Figure 2a);

- by applying a distributed force, deposited on the entire perimeter of the ring (Figure 2b).

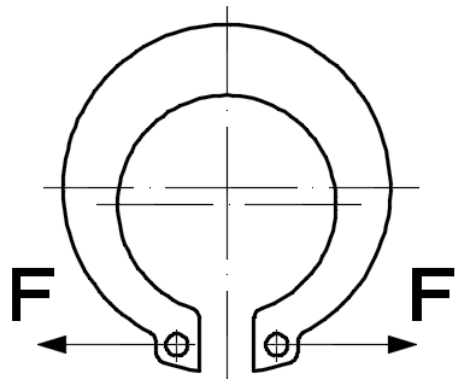

a)

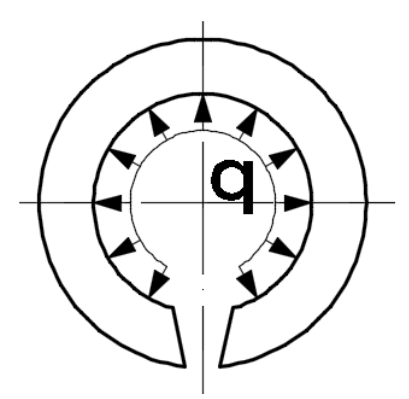

b)

Figure 2. Deformation methods for rings.

With concentrated forces, the rings with special holes at the ends are usually deformed; with distributed forces, by propelling on conical surfaces, the rings that do not have such holes are deformed. No information was found in the literature on the calculation of the forces required to deform the supporting rings. The joining of the supporting rings takes place after deformation, so the joining process and the permissible positioning errors are similar to those when joining the bushing with a shaft (Figure 3).

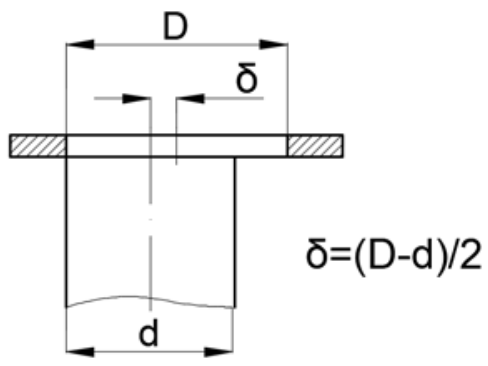

Figure 3. Permissible deviation when joining bushing to shaft.

It follows that a greater deformation of the supporting ring facilitates the joining, but its value should not exceed a certain allowable value, which would lead to damage to the supporting ring.

\section{Determination of the deformation forces of the supporting rings with rectangular transverse profile}

So, when joining the bearing rings are tightened or loosened, the difference being only in the direction of application of deformation forces.

Theoretically, eccentric rings in terms of deformation have more advantages than concentric ones. Due to their shape, the deformations in these rings are more evenly distributed, and, as will be shown below, such rings become more durable, especially at certain sizes. When mounting, the rings are deformed by the distributed force $q$ or by two concentrated forces $F$, deposited tangentially. When installed in the hole, the rings are tightened and on the shaft are loosed. Depending on this, the deformation forces are deposited in one direction or another, so the formulas that are applied to the rings installed 
on the shaft are equivalent to those that are applied to the rings installed in the hole, being different only the direction of forces and deformations.

The law of changing the cross section of the ring can be obtained in the following way: It follows from figure $4 \mathrm{a}$ that

$$
A B=r_{\min } \cdot \cos \beta_{1}
$$

and from the other part we have

$$
A B=r_{\max }-h-e \cdot \cos \varphi
$$

At small eccentricities the angle $\beta_{1}$ is small and it can be supposed that $\cos \beta_{1} \approx 1$. Connecting right-hand sides of equalities (1) and (2) we obtain:

$$
h=r_{\max }-r_{\min }-e \cdot \cos \varphi
$$

Let us denote the average hight of the ring as folows:

$$
r_{\max }-r_{\min }=\delta
$$

and denote also the relative eccentricity by:

$$
\frac{e}{\delta}=\chi
$$

Let it be the height of the cross section

$$
h=\delta \cdot(1-\chi \cdot \cos \varphi)
$$

The variation $\Delta_{\mathrm{j}}$ can be established as the difference of the lengths of the middle circumference before and after deformation of the ring:

$$
\Delta_{j}=2 \cdot \pi \cdot r 2-2 \cdot \pi \cdot r^{\prime} 2
$$

Within the limit of elastic deformations, the value $\Delta_{\mathrm{j}}$ is directly proportional to the intensity of the distributed force $q$ or concentrated force $F$. In both cases the proportionality coefficient between the given values can be found by means of the Mohr integral [12].

In order to do this we examine the half of the ring (Figures $4 b, 4 c$ ), considering one end of it as fixed and the other one as free.

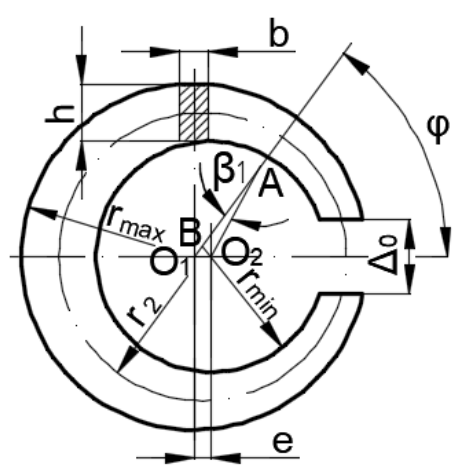

a)

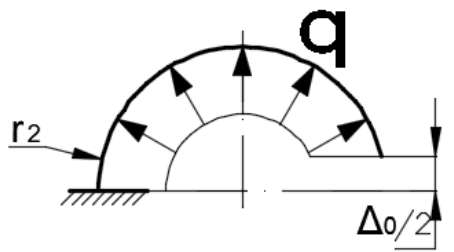

b)

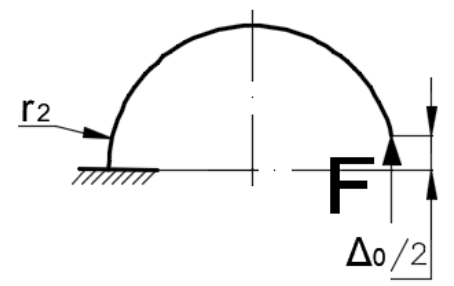

c)

Figure 4. The constructive scheme and formalization of deformation of supporting ring. 
The deformations of the second half of the ring will be simmetric to the deformations of the examined half of the ring.

Then in accordance to Mohr integral the move of the free end of the ring under the displacement of the free end from the force deposited tangent to the middle circumference of the ring will be:

$$
\frac{\Delta_{j}}{2}=\int_{0}^{\pi} \frac{M \cdot M_{1}}{E \cdot I} \cdot r_{2} d \varphi+\int_{0}^{\pi} \frac{k t r \cdot Q \cdot Q_{1}}{G \cdot F t r} \cdot r_{2} d \varphi+\int_{0}^{\pi} \frac{N \cdot N_{1}}{E \cdot F t r} r_{2} d \varphi
$$

where $r_{2}$ is the radius of the middle circumference of the ring;

$M, Q, N$ are functions of bending moment, transverse and normal forces, occurring, occurring in the cross section of the ring from the given force;

$M_{1}, Q_{1}, N_{1}$ are the same functions from the unit force deposited instead of the cut, tangent to the middle circumference;

$E, G$ is the modulus of elasticity of degree I and II of the material of the support ring;

$F_{t r}=b \cdot h$ is the cross-sectional area of the ring;

$I=\frac{b \cdot h^{3}}{12}$ curvature;

$k_{t r}=1.2$ is the coefficient, which depends on the transverse shape of the ring with a rectangular profile;

$\Delta_{j}=\Delta-\Delta_{0}$ is the change of play in the cut of the supporting ring, as a result of the actuation of the deformation force.

The functions of the internal factors are found using the section method. From the balance of the highlighted sector of the ring (Figure $4 b, 4 c$ ) we find:

- when the ring is deformed with distributed force q:

$$
\begin{aligned}
& M=-2 \cdot q \cdot r_{2}^{2} \cdot \sin ^{2} \frac{\varphi}{2} \\
& Q=-q \cdot r_{2} \cdot \sin \varphi \\
& N=2 \cdot q \cdot r_{2} \cdot \sin ^{2} \frac{\varphi}{2}
\end{aligned}
$$

- at the deformation of the ring with concentrated forces $\mathrm{F}$ :

$$
\begin{gathered}
M=-F \cdot r 2 \cdot(1-\cos \varphi) \\
Q=-F \cdot \sin \varphi \\
N=-F \cdot \cos \varphi
\end{gathered}
$$

At the deformation of the bar with unit force (Figure 5) in the given direction, from the equilibrium condition we have:

$$
\begin{gathered}
M_{1}=-r \cdot(1-\cos \varphi) \\
Q_{1}=-\sin \varphi \\
N_{1}=-\cos \varphi
\end{gathered}
$$




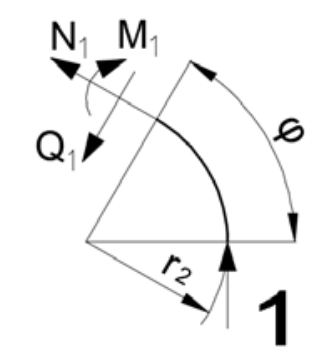

Figure 5. Deformation with unit force.

Substituting (9), (10), (11) in (8) and takinginto account the already known $F_{t r}, l, h$ we get:

a) when actuating the distributed force $q$ :

$$
\begin{aligned}
\frac{\Delta_{j}}{2}=\frac{24 \cdot q \cdot r_{2}^{4}}{E \cdot b \cdot \delta^{3}} \cdot \int_{0} \frac{\sin ^{2} \frac{\varphi}{2} \cdot(1-\cos \varphi) d \varphi}{(1-\chi \cos \varphi)^{3}}+\frac{1.2 \cdot q \cdot r_{2}^{2}}{G \cdot b \cdot \delta} \cdot \int_{0}^{\pi} \frac{\sin ^{2} \varphi d \varphi}{1-\chi \cdot \cos \varphi}- \\
-\frac{2 \cdot q \cdot r_{2}^{2}}{e \cdot b \cdot \delta} \cdot \int_{0}^{\pi \sin ^{2} \frac{\varphi}{2} \cdot \cos \varphi d \varphi} \frac{1-\chi \cdot \cos \varphi}{1-\chi}
\end{aligned}
$$

b) when actuating the concentrated force $F$ :

$$
\begin{gathered}
\frac{\Delta_{j}}{2}=\frac{12 \cdot F \cdot r_{2}^{3}}{E \cdot b \cdot \delta^{3}} \cdot \frac{\pi}{0} \frac{(1-\cos \varphi)^{2}}{0(1-\chi \cdot \cos \varphi)^{3}} d \varphi+\frac{1.2 \cdot F \cdot r 2}{G \cdot b \cdot \delta} \cdot \int_{0}^{\pi} \frac{\sin ^{2} \varphi}{1-\chi \cdot \cos \varphi} d \varphi+ \\
\quad+\frac{F \cdot r 2}{E \cdot b \cdot \delta} \cdot \int_{0}^{\pi} \frac{\cos ^{2} \varphi d \varphi}{1-\chi \cdot \cos \varphi}
\end{gathered}
$$

After integration the change of variation $\Delta$ is determined accordingly:

a)

$$
\begin{array}{r}
\Delta_{j}=\frac{36 \cdot \pi \cdot r_{2}^{4} \cdot q}{E \cdot b \cdot \delta^{3} \cdot\left(1+\chi^{2}\right) \cdot \sqrt{1-\chi^{2}}}+\frac{2.4 \cdot \pi \cdot q \cdot r_{2}^{2}}{G \cdot b \cdot \delta \cdot \chi^{2}} \cdot\left(1-\sqrt{1-\chi^{2}}\right)+ \\
+\frac{2 \cdot \pi \cdot q \cdot r^{2}}{E \cdot b \cdot \delta \cdot \chi^{2}} \cdot\left(\sqrt{\frac{1-\chi}{1+\chi}}+(\chi-1)\right)
\end{array}
$$

b)

$$
\begin{gathered}
\Delta_{j}=\frac{18 \cdot \pi \cdot F \cdot r_{2}{ }^{3}}{E \cdot b \cdot \delta^{3} \cdot\left(1+\chi^{2}\right) \cdot \sqrt{1-\chi^{2}}}+\frac{1.2 \cdot \pi \cdot F \cdot r_{2}}{G \cdot \delta \cdot b} \cdot \frac{1-\sqrt{1-\chi^{2}}}{\chi}+ \\
+\frac{\pi \cdot F \cdot r_{2}}{E \cdot b \cdot \delta \cdot \chi^{2}} \cdot\left(\frac{1}{\sqrt{1-\chi^{2}}}-1\right)
\end{gathered}
$$

In the rings used, the height of the cross section $h$ is much smaller than the radius of the middle circumference $r_{2}$. In this case the last two factors can be ignored without any major error for the accuracy of the calculation (the error does not exceed $0.3 \%$ ). 
Then:

$$
\begin{aligned}
& q=\frac{E \cdot b \cdot \delta^{3} \cdot\left(1+\chi^{2}\right) \cdot \sqrt{1-\chi^{2}}}{36 \cdot \pi \cdot r_{2}^{4}} \cdot \Delta_{j} \\
& F=\frac{E \cdot b \cdot \delta^{3} \cdot\left(1+\chi^{2}\right) \cdot \sqrt{1-\chi^{2}}}{18 \cdot \pi \cdot r^{3}} \cdot \Delta_{j}
\end{aligned}
$$

Thus knowing the change of the variation $\Delta$, from expressions (14) and (15) we can calculate the forces $q$ and $F$, which will not exceed the permissible ones, which in turn depend on the durability of the ring:

$$
\sigma_{\max }=\frac{M_{\max }}{W}=\frac{6 \cdot q \cdot r_{2}^{2} \cdot(1-\cos \varphi)}{b \cdot \delta^{2} \cdot(1-\chi \cdot \cos \varphi)^{2}} \leq[\sigma]
$$

or

$$
\sigma_{\max }=\frac{M_{\max }}{W}=\frac{6 \cdot F \cdot r_{2} \cdot(1-\cos \varphi)}{b \cdot \delta^{2} \cdot(1-\chi \cdot \cos \varphi)^{2}} \leq[\sigma]
$$

where $M_{\max }$ is the moment of bending in the dangerous section of the ring;

$\mathrm{W}$ is the moment to oppose bending in this section;

$[\sigma]$ are allowable stresses of the ring material.

The optimization of the dimensions of the support rings consists in determining the eccentricity $\chi$ at which the change of value $\Delta$ would be maximum. At the same time, changing the value of $\chi$ changes the place of the dangerous section in the ring. At low values of $\chi$ it is located opposite to the cut. With increasing $\chi$ the place of the dangerous section approaches the cut.

For this reason, when optimizing the dimensions of the support rings, first of all the angle $\phi$ at which the dangerous section in the ring is located must be determined and only then the dependence of this angle on the eccentricity $\chi$.

In the dangerous section the stresses are maximum, therefore to find the place of this section it is necessary to study the extremes of the function $\sigma_{\max }$ :

$$
\frac{d \sigma_{\max }}{d \varphi}=\frac{6 \cdot q \cdot r_{2}^{2}}{b \cdot \delta^{2}} \cdot \sin \varphi \cdot \frac{(1-\chi \cdot \cos \varphi)-2 \cdot \chi \cdot(1-\cos \varphi)}{(1-\chi \cdot \cos \varphi)^{3}}=0
$$

or

$$
\frac{d \sigma_{\max }}{d \varphi}=\frac{6 \cdot F \cdot r_{2}}{b \cdot \delta^{2}} \cdot \sin \varphi \cdot \frac{(1-\chi \cdot \cos \varphi)-2 \cdot \chi \cdot(1-\cos \varphi)}{(1-\chi \cdot \cos \varphi)^{3}}=0
$$

Equality to zero of the derivative of the function $\sigma_{\max }$ is possible if $\sin \phi=0$ or:

$$
\frac{(1-\chi \cdot \cos \phi)-2 \cdot \pi \cdot(1-\cos \phi)}{(1-\chi \cdot \cos \phi)^{3}}=0
$$

In the first case, the dangerous section is located opposite the cut and does not depend on the eccentricity $\chi$, corresponding to rings with a constant profile $(\chi=0)$. 
For rings with variable profile, formula (22) enters into force, which represents a transcendent equation with respect to the variable $\phi$. The solution ofthis equation in the interval $\chi=0 \ldots 1$ with step 0.01 is represented in Figure 6, from which follows that the dangerous section is located in the plane of symmetry of the ring $\left(\phi=180^{\circ}\right)$ at $\chi=0 . . .0 .33$.

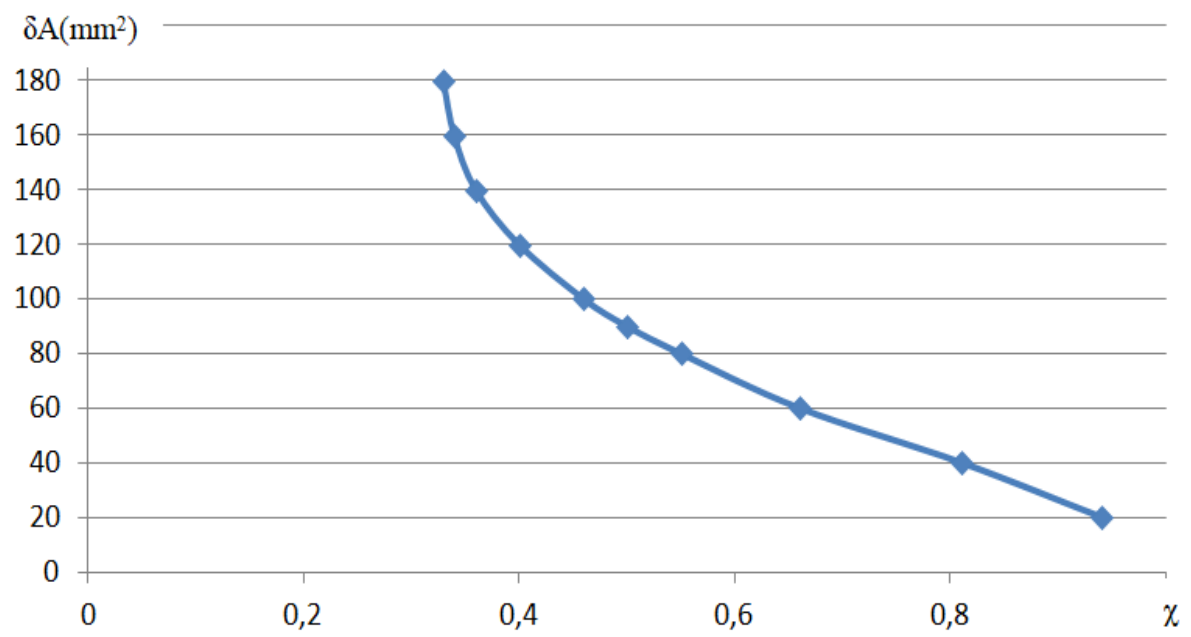

Figure 6. Location angle of the dangerous section $\phi^{\circ}$ depending on the eccentricity $\chi$.

Increasing the eccentricity moves the dangerous section to the cut of the ring. At $\chi=1 \phi$ tends to zero.

The change of the eccentricity also leads to the change of the values of the forces necessary for the deformation and the maximum value of the variation $\Delta_{\mathrm{j}}$, respecting the condition of avoiding the plastic deformations of the deformed ring. These changes can be calculated by the formulas $(16,17,18,19)$. Figures 7,8 show the graphs obtained as a result of the calculation of the change $F$ and $\Delta_{\mathrm{j}}$ depending on the change of eccentricity $\chi$ for rings with the dimensions:

$$
\begin{aligned}
& r_{2}=24.3 ; \\
& b=1.5 ; \\
& \delta=4.1 ; \\
& {[\sigma]=240 \mathrm{Mpa} ;}
\end{aligned}
$$

And step $x=0.01$ in the interval from 0 to 1 .

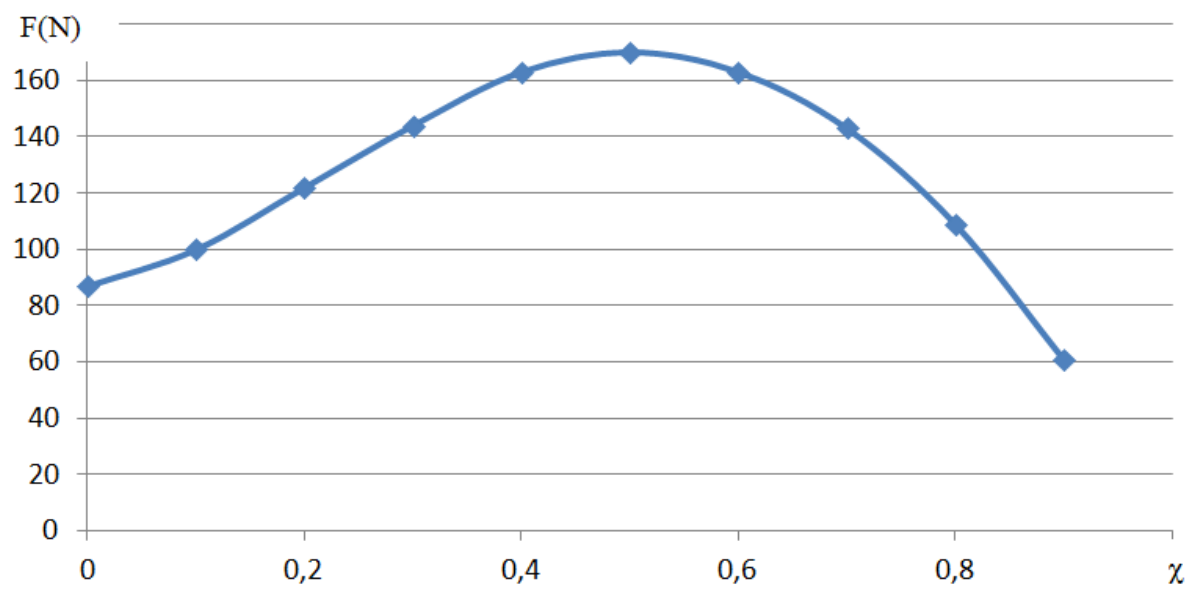

Figure 7. Dependence of the allowable force applied to the support ring $F(N)$ as a function of eccentricity $x$. 


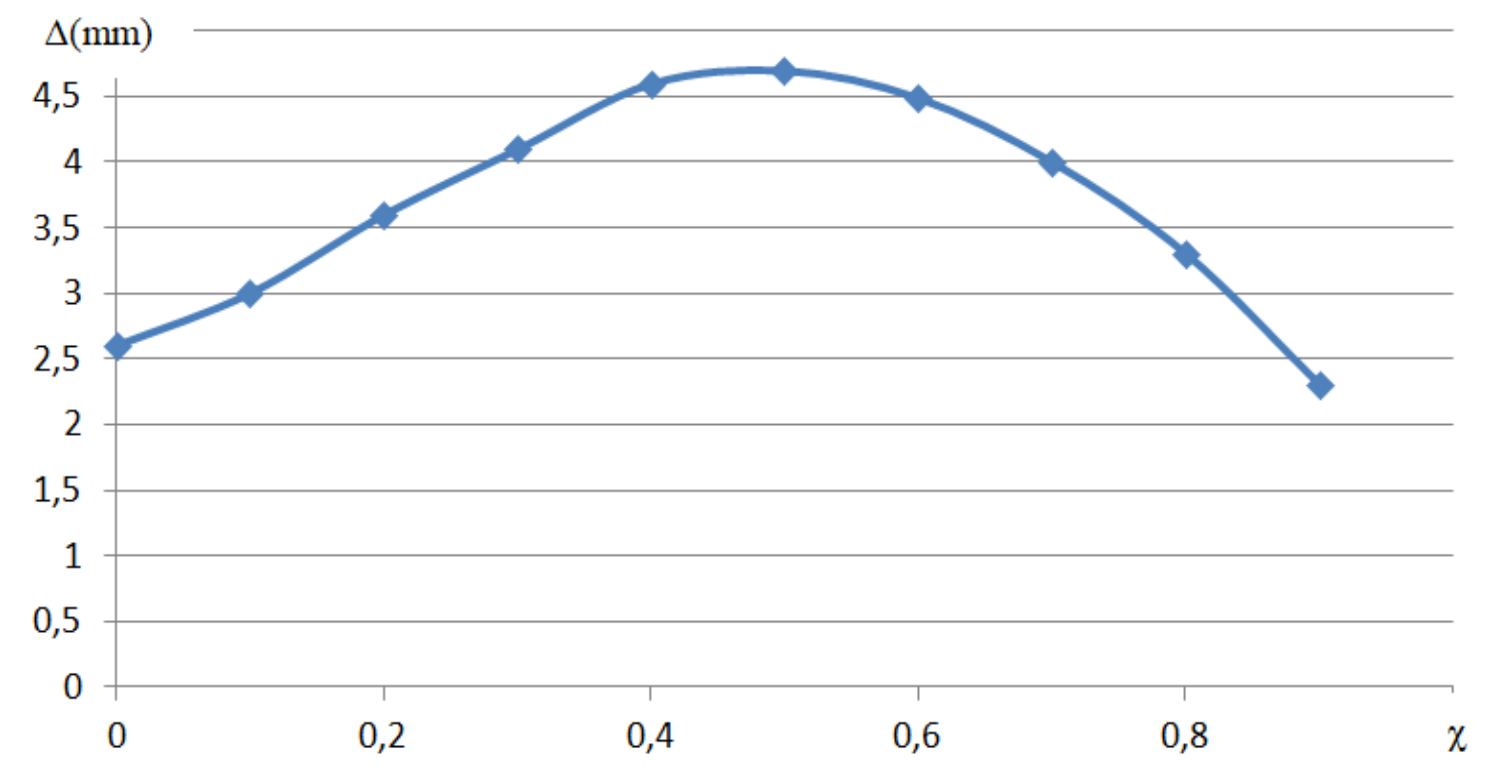

Figure 8. Dependence of the permissible deformation of the support ring $\Delta(\mathrm{mm})$ as a function of eccentricity $x$.

The initial parameters of the supporting rings (sizes and material) do not influence the change of their deformation parameters; optimal eccentricity $\chi=0.49$, at which the permissible deformation acquires maximum values remains unchanged.

\section{Optimizing the eccentricity of the rings and the angle of application of the deformation forces}

The rings are deformed by the forces $F$, directed at a certain angle a to the tangents to the outer circumference of the ring (Figure 9). The change in the surface of the circle with radius $r_{2}$ can be taken as a parameter of the deformation of the rings.

When deforming, the ring deviates from the round shape, but this deviation can be neglected, being a variable value and difficult to appreciate.

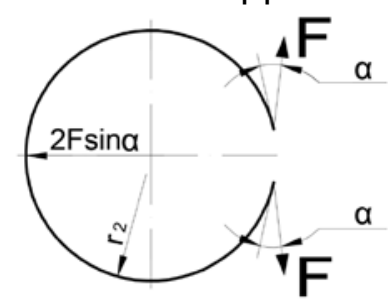

Figure 9. The scheme of application of deformation forces.

Mohr's integral for changing the surface of the circle with radius $r_{2}$ :

$$
\delta A=2 \cdot \int_{0}^{\pi} \frac{M_{F} \cdot M_{1}}{E \cdot I} d \phi+2 \cdot \int_{0}^{\pi} \frac{N_{F} \cdot N_{1}}{E \cdot F_{t r}} d \phi+2 \cdot \int_{0}^{\pi} \frac{k_{t r} \cdot Q_{F} \cdot Q_{1}}{G \cdot F_{t r}} d \phi
$$

where $F_{t r}=b h$ is the cross-sectional area of the considered ring;

$I=b h^{3} / 2-$ is the moment of inertia of the cross section;

$R_{t r}$ - is the coefficient of the transverse shape of the ring.

Functions of internal factors occurring when actioning the force $F: M_{F}, N_{F}$ and $Q_{F}$ or by actioning the force $q: M_{1}, N_{1}$ and $Q_{1}$ are determined by the method of sections (Figure 10): 

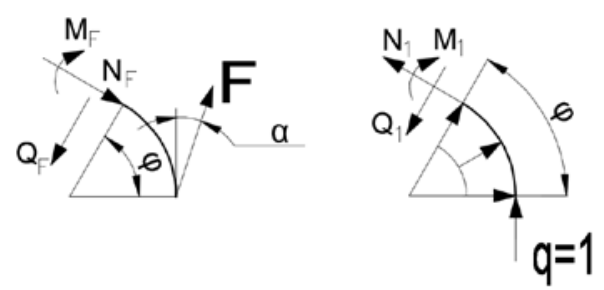

Figure 10. Formalization of the support ring deformation.

$$
\begin{gathered}
M_{F}=-F \cdot r_{2} \cdot \cos \alpha \cdot(1-\cos \phi)-F \cdot r_{2} \cdot \sin \phi \cdot \sin \phi \\
N_{F}=-F \cdot \cos \alpha \cos \phi+F \cdot \sin \alpha \cdot \sin \phi ; \\
Q_{F}=-F \cdot \cos \alpha \cdot \sin \phi-F \cdot \sin \alpha \cdot \cos \phi \\
M_{1}=-r_{2}^{2} \cdot(1-\cos \phi) ; \\
N_{1}=r_{2} \cdot(1-\cos \phi) ; \\
Q_{1}=-r_{2} \cdot \sin \phi .
\end{gathered}
$$

Substituting the above values in (23) we get:

$$
\begin{gathered}
\delta A=24 \int_{0}^{\pi F \cdot \cos \alpha \cdot r_{2}^{4} \cdot\left(1-\cos \varphi^{2}\right)} \frac{E \cdot b \cdot \delta^{3}(1-\chi \cdot \cos \varphi)^{3}}{0} d \varphi- \\
2 \cdot \int_{0}^{\pi} \frac{F \cdot \cos \alpha \cdot r_{2}^{2} \cdot \cos \phi \cdot(1-\cos \phi)}{E \cdot b \cdot \delta^{3} \cdot(1-\chi \cdot \cos \phi)} d \phi+ \\
+2.4 \int_{0}^{\pi} \frac{F \cdot \cos \alpha \cdot r_{2}^{2} \cdot \sin ^{2} \varphi}{G \cdot b \cdot \delta \cdot(1-\chi \cdot \cos \varphi)} d \varphi+24 \int_{0} \frac{\pi \cdot \sin \alpha \cdot r_{2}^{4} \cdot \sin \varphi \cdot(1-\cos \varphi)}{E \cdot b \cdot \delta^{3} \cdot(1-\chi \cdot \cos \varphi)^{3}} d \varphi+ \\
+2 \cdot \int_{0}^{\pi} \frac{F \cdot \sin \alpha \cdot r_{2}^{2} \cdot \sin \phi \cdot(1-\cos \phi)}{E \cdot b \cdot \delta \cdot(1-\chi \cdot \cos \phi)} d+2.4 \cdot \int_{0}^{\pi} \frac{F \cdot \sin \alpha \cdot r_{2}^{2} \sin \phi \cdot \cos \phi}{G \cdot b \cdot \delta \cdot(1-\chi \cdot \cos \phi)} d \phi
\end{gathered}
$$

and after integration we come to:

$$
\begin{gathered}
\delta A=\frac{2 \cdot F \cdot \cos \alpha \cdot \pi \cdot r_{2}^{2}}{b \cdot \delta} \frac{18 \cdot r_{2}^{2}}{E \cdot \delta^{2}} \cdot\left[\frac{18 \cdot r_{2}^{2}}{E \cdot \delta^{2}} \cdot \frac{1}{\sqrt{1-\chi^{2}} \cdot(1+\chi)^{2}}+\frac{1}{E \cdot \chi^{2}} \cdot\left(\sqrt{\frac{1-\chi}{1+\chi}}-(1+\chi)\right)+\right. \\
\left.+\frac{1,2}{G \cdot \chi^{2}} \cdot\left(1-\sqrt{1-\chi^{2}}\right)-\right]+\frac{4 \cdot F \cdot \sin \alpha \cdot r_{2}^{2}}{b \cdot \delta} \cdot\left[\frac{12 \cdot r_{2}^{2} \cdot(1-\chi)}{E \cdot \delta^{2} \cdot\left(1-\chi^{2}\right)}+\frac{1}{E \cdot \chi^{2}} \cdot\left(\chi+\frac{1-\chi}{2} \cdot \ln \frac{1-\chi}{1+\chi}\right)-\right. \\
\left.-\frac{1.2}{G \cdot \chi^{2}} \cdot\left(\chi+\frac{1}{2} \ln \frac{1-\chi}{1+\chi}\right)\right]
\end{gathered}
$$

This formula allows us to establish the influence of angle $\alpha$ and eccentricity $\chi$ on the deformation of the ring. The first factors in the square bracket play a key role in the formula. The rest of the factors can be neglected without losing the accuracy of the calculations.

Maximum elastic deformations of the ring, beware:

$$
\sigma_{\max }=\frac{F \cdot r_{2} \cdot(\cos \alpha \cdot \cos \varphi)}{b \cdot h \cdot e_{1}} \cdot \frac{h / 2-e_{1}}{r_{2}-h / 2}
$$


where $e_{1}$ is the eccentricity of the neutral line:

$$
e_{1}=h^{2} / 12 \cdot r_{2}
$$

Then:

$$
\sigma_{\max }=\frac{2 \cdot F \cdot r_{2}}{b} \cdot \frac{\cos \alpha-\cos \varphi}{\delta^{2} \cdot(1-\chi \cdot \cos \varphi)^{2}} \cdot \frac{6 \cdot r_{2} \cdot(1-\chi \cdot \cos \varphi)}{2 \cdot r-\delta \cdot(1-\chi \cdot \cos \varphi)}
$$

The given formula suppose the knowledge of the angle $\phi$, which depends on the angle $\alpha$ and the eccentricity $\chi$.

If $\alpha$ and $\chi$ are known, the place of the dangerous section is calculated by studying the extremes of the function:

$$
\begin{gathered}
\frac{d \sigma_{\max }}{d \varphi}=\frac{2 \cdot F \cdot r_{2}}{b \cdot \delta^{2}} \cdot \sin \varphi \cdot\left[\frac{(1-\chi \cdot \cos \varphi)-2 \cdot \chi \cdot(\cos \alpha-\cos \varphi)}{(1-\chi \cdot \cos \varphi)^{3}} .\right. \\
\left.\frac{6 \cdot r_{2}-\delta \cdot(1-\chi \cdot \cos \varphi)}{2 \cdot r-\delta \cdot(1-\chi \cdot \cos \varphi)}+\frac{\cos \alpha-\cos \varphi}{(1-\chi \cdot \cos \varphi)^{2}} \cdot \frac{4 \cdot r_{2} \cdot \delta \cdot \chi}{(2 \cdot r-\delta \cdot(1-\chi \cdot \cos \varphi))^{2}}\right]=0
\end{gathered}
$$

This equality can take place for $\sin \varphi=0$ or when the value of thesquare paranthesis is equal to zero. $\mathrm{n}$ the first case, the dangerous section is located opposite the ring cut $\left(\phi=180^{\circ}\right)$ and correponds to equality to zero of a and $\chi$.

In the second case, the place of the dangerous section is found by solving the transcendental equation (in square brackets), equaling it with zero.

Thus the algorithm for studying the dependence of the ring deformation on the angle a and the eccentricity $\chi$ contains three stages:

1. Determining the angle $\phi$ having a and $\chi$ known;

2. Calculation of the maximum allowable force required for the deformation of the ring, based on the condition $\sigma_{\max }\langle[\sigma]$;

3. Determination of the parameter $\delta A$ by changing $a$ and $\chi$.

In order to automate the process of optimizing the dimensions of the rings and determining the optimal angle a of actuation of the deformation forces, formulas 26, 28 and 29 were algorithmized, formula 29 being calculated by Ribacov's iteration method [13], because it is unsolvable mathematically.

The results of the calculations for the supporting rings with the parameters:

$r_{2}=24.3$;

$b=1.5$;

$\delta=4.1$

$[\sigma]=240 \mathrm{Mpa}$;

are presented in the Figures 11 and 12. 


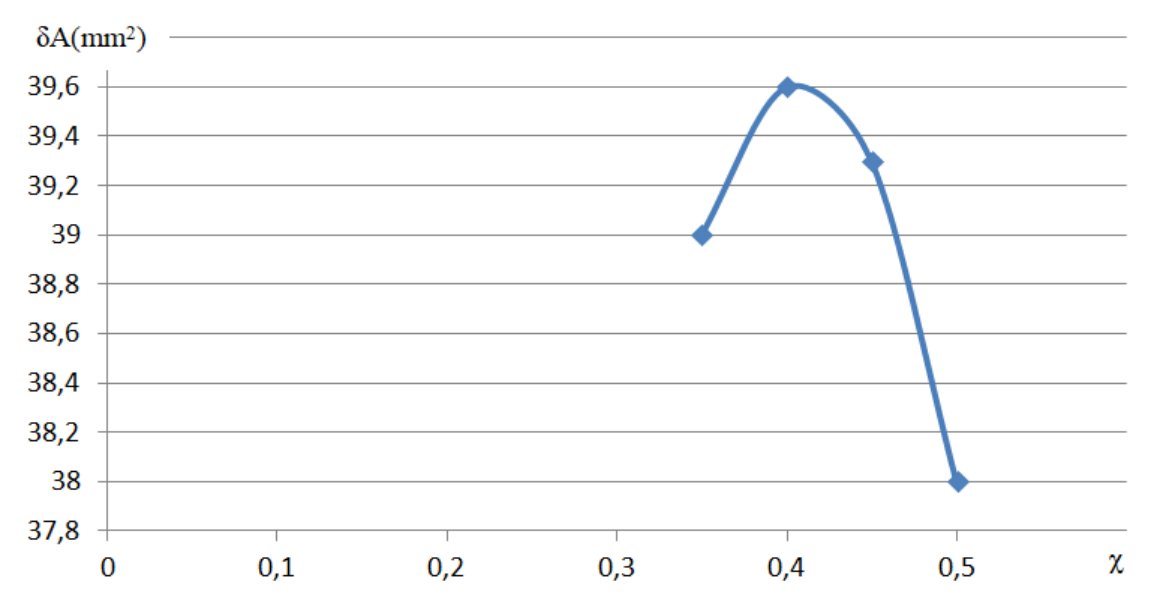

Figure 11. Dependence of the allowable deformation of the supporting ring $\delta \mathrm{A}\left(\mathrm{mm}^{2}\right)$ as a function of eccentricity $\chi$ with forces applied at an angle $\alpha=0^{\circ}$.

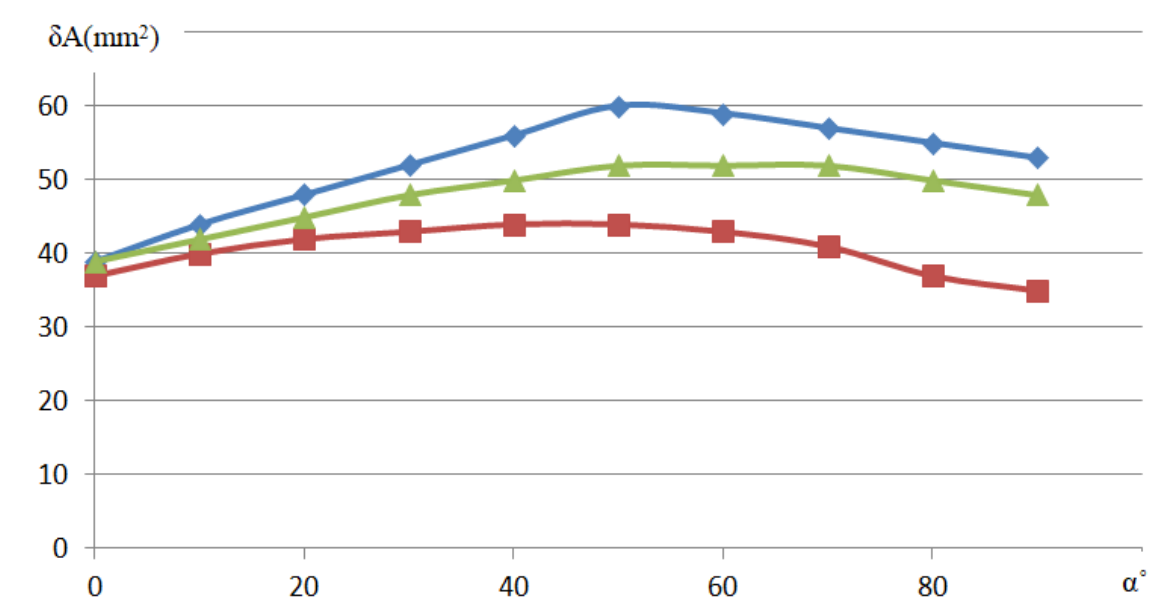

Figure 12. Dependence of the allowable deformation of the supporting ring $\delta A\left(\mathrm{~mm}^{2}\right)$ depending on the angle of application $\alpha$ of the deformation forces for eccentricit $\chi=0$ ( $g$ raph with symbol $\square$ ), $\chi=0,33$ (graph with symbol $\Delta$ ), $\chi=0,4$ (graph with symbol $\diamond$ ).

\section{Conclusions}

1) Eccentric rings create more pleasant conditions for deformation, due to the uneven location of the material in them;

2) The rings with the relative eccentricity 0.5 , which was determined as optimal, possess an increased deformability by 20 ... 25\% compared to those stipulated in the standards GOST 13942-83 and GOST 13943-86, which are executed with the relative eccentricity 0.2. ..0.3;

3) The tightening and unwinding processes of the rings are equivalent, the calculation formulas in both cases are the same, only the deformation forces changing their sign;

4) When applying the deformation forces at an angle of 50 degrees, determined as optimal, the deformability of the eccentric rings increases by $25 \ldots 30 \%$ and of the concentric ones by about 20\%;

5) The value of the optimal relative eccentricity is not influenced by the dimensions and the material of the rings, always remaining constant. 


\section{References}

1. Jonson E. Automating assembly-A crucial step for day one. Assemblymag.com. 2020, November 5. https://www.assemblymag.com/articles/95980-automating-assemblya-crucial-step-for-day-one - accessed 20.07.2021.

2. Abramova L.D., Salomatin N.A. Modern problems of production automation. University Bulletin, 2012, 7, pp. 42-49, ISSN 1816-4277 [in Russian].

3. Bozhko A.N. Automation of the design of assembly processes of complex products using virtual reality. Intelligent IT in management, 2018, N2, pp. 36-40, UDC.67.02, 004.942, 519 [in Russian].

4. Semenov A.N. Manufacturability of the design of the mechanical engineering product. Rybinsk, RGTAU, 2016, 217r., UDC.621 [in Russian].

5. https://studme.org/97106/tehnika/metodika_posledovatelnost_proektirovaniya_tehnologicheskih_protsesso v_sborki- accessed 20.07.2021.

6. Vorobiev E. I., Gavryushin S. S. New mechanisms in modern robotics. Moscow, Technosphere, 2018, 316p., ISBN: 978-5-94836-537-4.178 [in Russian].

7. Lorre B. L'automatisation industrielle, quel avenir pour les usines du futur?, 22.10.2020, iatranshumanisme.com- accessed 02.02.2021.

8. Costa M. J. R., Gouveia R. M., G. Silva F. J., Campilho R. D. S. How to Solve quality problems by advanced fullyautomated manufacturing systems. The international journal of Advanced Manufacturing Technology, February 2018, 94(1), pp. 3041-3063, DOI: 10.1007/S00170-017-0158-8.

9. GOST 27017-86 (ISO 1891-79) Fasteners. Terms and Definitions [in Russian].

10. http://www.smalley.ru/stati/stopornye-kolca- accessed 20.07.2021.

11.http://sibindustry.ru/engineering/stopornyje-kolca-vidy-kharaktjeristiki-sfjera-primjenjenija.html- accessed 20.07.2021.

12. Feodosiev V.I. Resistance of materials. Moscow, MSTU im. N.E.Bauman, 2018, 544p., ISBN: 978-5-70384819-7 [in Russian].

13.Zhavoronkov L. Numerical Analysis in Pascal ABC: Studies in Applied Mathematics. Amazon.com Services LLC, 2020, 409p., ISBN: 978-5-906886-71-2. 